\title{
Establishing HZ43 A, Sirius B, and RX J185635-3754 as soft X-ray standards: a cross-calibration between the Chandra LETG+HRC-S,

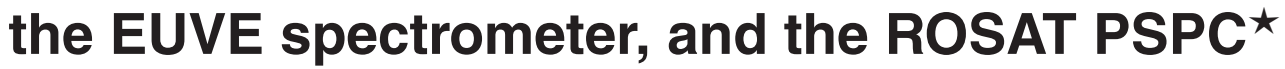

\author{
K. Beuermann ${ }^{1}$, V. Burwitz ${ }^{2}$, and T. Rauch ${ }^{3}$ \\ 1 Institut für Astrophysik, Friedrich-Hund-Platz 1, 37077 Göttingen, Germany \\ e-mail: beuermann@astro.physik.uni-goettingen.de \\ 2 MPI für Extraterrestrische Physik, 85748 Garching, Germany \\ e-mail: burwitz@mpe.mpg.de \\ 3 Institut für Astronomie und Astrophysik, Sand 1, 72076 Tübingen, Germany \\ e-mail: rauch@astro.uni-tuebingen.de \\ A\&A 458, 541-552 (2006) DOI: 10.1051/0004-6361:20065478
}

Key words. errata, addenda - methods: data analysis - stars: white dwarfs - stars: neutron - stars: fundamental parameters

Jelle Kaastra (private communication) noted differences between the incident soft X-ray spectrum of Sirius B given in Table 3 of Beuermann et al. (2006) and his own version, whereas the similarly derived HZ43 A spectra agree excellently. The differences are caused by two trivial errors in our paper and by a non-trivial systematic difference in the model atmosphere spectra created with the TLUSTY code used by Kaastra and TMAP used by us.

Table 2 erroneously quotes the parameters of the simultaneous fit to the LETG+HRC spectra of HZ43 A, Sirius B, and RXJ1856-37 for $\log g($ Sirius B $)=8.55$ instead of the preferred $\log g=8.60$. The latter gives $T_{\text {eff }}=24897 \pm 115 \mathrm{~K}$ for Sirius B and $T_{\text {eff }}=51111 \pm 660 \mathrm{~K}$ for HZ43 A, which is only $26 \mathrm{~K}$ and $15 \mathrm{~K}$ lower than for $\log g=8.55$. All other parameters of the simultaneous fit change only by a minute $0.03-0.11 \%$. Table 3 incorrectly gives the Sirius B fluxes without interstellar absorption, which raises them noticeably at longer wavelengths. We enclose the revised table for $\log g=8.60$ (Sirius B) and $\log g=7.90$ (HZ43 A). For $\log g=8.55$, the fluxes of Sirius B at 60,100 , and $160 \AA$ change by $-1.4 \%,+0.2 \%$, and $-0.2 \%$, respectively. None of our conclusions is affected.

The non-trivial difference in the model fluxes results from different assumptions on the level dissolution by pressure ionization in TLUSTY and TMAP. Level dissolution is unimportant in the hot atmosphere of HZ43 A explaining why the discrepancy only exists for Sirius B. The unsolved problem of level dissolution is discussed by Rauch (2008).

\section{References}

Beuermann, K., Burwitz, V., \& Rauch, T. 2006, A\&A, 458, 541 Rauch, T. 2008, A\&A, 481, 807

* Revised versions of Tables A.1-A.6 are only available electronically at

http://cdsweb.u-strasbg.fr/cgi-bin/qcat?J/A+A/458/541
Table 3. Soft X-ray fluxes of HZ43 A, Sirius B, and RXJ1856-37 at the top of the atmosphere. The numbers in brackets are the systematic errors of the LETG+HRC effective area correction in percent.

\begin{tabular}{|c|c|c|c|}
\hline $\begin{array}{l}\lambda \\
(\AA)\end{array}$ & HZ43 A & $\begin{array}{c}\text { Sirius B } \\
\text { photons } \mathrm{cm}^{-2} \mathrm{~s}^{-1} \AA^{-1}\end{array}$ & RX J1856 \\
\hline $\begin{array}{r}20 \\
30 \\
40 \\
43 \\
44 \\
48 \\
60 \\
70 \\
80 \\
90 \\
100 \\
125 \\
160\end{array}$ & $\begin{array}{ll}3.00 \times 10^{-4} & (5)^{1)} \\
2.13 \times 10^{-3} & (5) \\
6.32 \times 10^{-3} & (5) \\
1.39 \times 10^{-2} & (6) \\
2.49 \times 10^{-2} & (6) \\
3.88 \times 10^{-2} & (6) \\
7.95 \times 10^{-2} & (7) \\
1.31 \times 10^{-1} & (7)\end{array}$ & $\begin{array}{l}2.45 \times 10^{-4}(6) \\
9.38 \times 10^{-4}(6) \\
2.52 \times 10^{-3}(6) \\
5.30 \times 10^{-3}(6) \\
9.20 \times 10^{-3}(6) \\
2.01 \times 10^{-2}(7) \\
2.37 \times 10^{-2}\end{array}$ & $\begin{array}{ll}8.53 \times 10^{-5} & (5) \\
4.32 \times 10^{-4} & (5) \\
6.54 \times 10^{-4} & (5) \\
6.70 \times 10^{-4} & (5) \\
6.94 \times 10^{-4} & (5) \\
6.80 \times 10^{-4} & (5) \\
5.05 \times 10^{-4} & (7) \\
3.10 \times 10^{-4} & (7)\end{array}$ \\
\hline & \multicolumn{3}{|c|}{$\operatorname{erg~cm}{ }^{-2} \mathrm{~s}^{-1} \AA^{-1}$} \\
\hline $\begin{array}{l}1300 \\
4600 \\
5000 \\
5450\end{array}$ & $4.052 \times 10^{-12}$ & $\begin{array}{l}1.253 \times 10^{-10} \\
2.855 \times 10^{-12}\end{array}$ & $\begin{array}{l}5.07 \times 10^{-172)} \\
2.96 \times 10^{-193)}\end{array}$ \\
\hline
\end{tabular}

1) The Compton effect may depress this flux by $\sim 1 \%$.

2) The unabsorbed flux is $6.27 \times 10^{-17} \mathrm{erg} \mathrm{cm}^{-2} \mathrm{~s}^{-1} \AA^{-1}$.

3) The unabsorbed flux is $3.18 \times 10^{-19} \mathrm{erg} \mathrm{cm}^{-2} \mathrm{~s}^{-1} \AA^{-1}$. 